\title{
Recurrence of Hepatocellular Carcinoma in Hepatitis C Virus (HCV) Liver Transplant Recipients Treated with Pretransplant Direct-Acting Antiviral (DAA) Therapy
}

\author{
Nicholas Lim ${ }^{a}$ Dupinder Singh ${ }^{a}$ Scott Jackson ${ }^{b}$ John R. Lake \\ aDivision of Gastroenterology, Hepatology and Nutrition, Department of Medicine, \\ University of Minnesota, Minneapolis, MN, USA; bFairview Health Services, Minneapolis, \\ MN, USA
}

\section{Keywords}

Hepatitis C · Direct-acting antiviral - Liver transplant · Hepatocellular carcinoma - Recurrence

\begin{abstract}
Background: Direct-acting antivirals (DAAs) have revolutionized the treatment of hepatitis $C$ virus (HCV). The impact of DAAs on recurrence of hepatocellular carcinoma (HCC) after liver transplantation (LT) remains uncertain. Objective: We aimed to evaluate the risk of HCC recurrence in LT recipients cleared of HCV with DAAs at the time of LT compared to a control group of LT recipients who were viremic at the time of LT. Methods: The study was a singlecenter, retrospective cohort study of patients undergoing LT for HCV-related HCC from 2002 to 2017. We compared time to post-LT HCC recurrence in patients with a sustained virological response (SVR) from DAAs prior to LT (DAA group) to patients who were viremic at LT (HCV+ group) using Kaplan-Meier analysis. We performed a secondary analysis comparing post-LT HCC recurrence in the DAA group to LT recipients with SVR from interferon-based treatment prior to LT (IFN group). Results: One hundred fifty-one patients underwent LT for HCC related to HCV: 34 patients in DAA group, 95 patients in HCV+ group, and 22 in IFN group. Kaplan-Meier estimates of being HCC free were $96.2,96.2$, and $78.8 \%$ at 6,12 , and 24 months in DAA group, respectively, and $100,98.6$, and $95.8 \%$ at 6,12 , and 24 months in the HCV+ group, respectively; $p=0.08$. There was no difference observed for HCC recurrence between the DAA and IFN groups. In a multivariate Cox proportional hazards model, DAA use increased the risk of post-LT HCC recurrence (HR 5.2, 95\% Cl 0.9-29.81, $p=0.07$ ). Conclusions: A strong
\end{abstract}


trend was observed on both Kaplan-Meier and multivariate analyses toward increased postLT HCC recurrence in patients who achieved SVR prior to LT with DAAs compared to patients who were viremic at LT. Caution is required when considering pre-LT treatment of HCV with DAAs in patients with HCC.

(c) 2020 The Author(s)

Published by S. Karger AG, Basel

\section{Introduction}

Hepatocellular carcinoma (HCC) is a leading cause of cancer-related mortality worldwide [1]. Liver transplantation (LT) for HCC is curative for cirrhosis and offers excellent long-term overall and cancer-free survival in patients within Milan criteria [2]. Organ Procurement and Transplantation Network data show that $14.4 \%$ of all liver transplants performed in 2016 were for a primary diagnosis of HCC [3].

Hepatitis $\mathrm{C}$ virus (HCV) infection is the most common cause of cirrhosis and HCC in the Western world [4-6]. Direct-acting antiviral (DAA) therapy has revolutionized the management of HCV by markedly improving efficacy with few side effects as compared to interferon-based treatment [7-10]. In LT, the use of DAAs pre- or post-LT has reduced posttransplant recurrence of HCV and has improved posttransplant graft and patient survival [11-13].

Recent reports have reached differing conclusions regarding the occurrence and recurrence of HCC in patients with sustained virological response (SVR) after treatment with DAAs. Two studies have reported higher rates of de novo or recurrent HCC in patients with SVR after DAAs undergoing locoregional therapy (LRT) or resection for HCC $[14,15]$. In contrast, other reports have shown no difference in recurrence of HCC in patients treated with DAAs after LRT on the LT waitlist $[16,17]$. A recent large multicenter study concluded that DAA therapy was not associated with HCC recurrence in patients treated after complete response to a variety of therapies [18]. To date, only 1 brief report has evaluated recurrence of HCC in LT recipients with HCV after treatment with DAAs, which showed a higher rate of HCC recurrence in patients who achieved SVR with DAAs, which did not reach statistical significance [19]. We evaluated the risk of HCC recurrence in HCV+ LT recipients with SVR after treatment with DAAs prior to LT compared to a control group transplanted for HCC and HCV who remained viremic at the time of LT.

\section{Materials and Methods}

Patients

We conducted a single-center, retrospective cohort study of all patients undergoing LT for HCC in $\mathrm{HCV}(+)$ recipients from 2002 to 2017. Data were collected using the Transplant Information Services at our institution. Data unable to be acquired this way were obtained through manual chart review with approval of the local Institutional Review Board. Patients were excluded if they were $<18$ years of age, outside Milan criteria for LT for HCC at the time of LT, underwent re-transplant, had a history of hepatitis B infection or other concurrent liver disease, if they achieved SVR with boceprevir or telaprevir, or if the medical record was incomplete. Data regarding demographics, clinical features, and HCC characteristics were collected. Tumor factors studied included size at diagnosis and just before transplant, number of lesions, treatment received, and explant pathology.

\section{Outcomes}

The primary outcome of this study was HCC recurrence post-LT based on standard imaging criteria or biopsy. Subjects were divided into 2 groups: (1) patients who achieved SVR12 from DAAs prior to LT (DAA group), and (2) patients who were either not treated or did not achieve SVR12 (HCV+ group). HCC-free 
Table 1. Baseline characteristics ( $n(\%)$ or median $[\mathrm{IQR}]$ )

\begin{tabular}{lccr}
\hline & DAA group $(n=34)$ & HCV+ group $(n=95)$ & $p$ value \\
\hline Age at transplant, years & $60.2[58.1 ; 62.5]$ & $57.2[53.1 ; 61.6]$ & $<0.01$ \\
Gender, $n$ (\%) & $6(17.6)$ & $11(11.6)$ & 0.38 \\
$\quad$ Female & $28(82.4)$ & $84(88.4)$ & \\
$\quad$ Male & $25(73.5)$ & $74(77.9)$ & 0.78 \\
White race, $n$ (\%) & $9(26.5)$ & $19(20.0)$ & 0.59 \\
Diabetes mellitus, $n$ (\%) & $34(100)$ & $78(82.1)$ & 0.01 \\
Deceased donor LT, $n$ (\%) & $9.00[8.00 ; 13.5]$ & $13.0[10.0 ; 17.5]$ & $<0.01$ \\
MELD score at transplant & $1.16[0.75 ; 1.38]$ & $0.58[0.38 ; 1.21]$ & $<0.01$ \\
Wait-list time, years & $0.80[0.60 ; 1.62]$ & $1.40[0.80 ; 2.40]$ & 0.04 \\
Total bilirubin, mg/dL & $32.0[25.0 ; 57.0]$ & $64.0[40.0 ; 110]$ & $<0.01$ \\
ALT, U/L & $36.0[25.0 ; 53.2]$ & $86.0[62.8 ; 130]$ & $<0.01$ \\
AST, U/L & $106[89.2 ; 132]$ & $138[95.0 ; 194]$ & 0.01 \\
Alkaline phosphatase, U/L & $3.50[3.10 ; 3.80]$ & $3.15[2.70 ; 3.50]$ & 0.01 \\
Albumin, g/dL & $8.15[5.20 ; 13.3]$ & $22.3[9.38 ; 74.9]$ & $<0.01$ \\
AFP, ng/dL & $5.75[3.92 ; 6.75]$ & $5.30[3.90 ; 7.10]$ & 0.88 \\
WBC $\left(\times 10^{9} / \mathrm{L}\right)$ & $13.0[11.5 ; 14.2]$ & $12.7[11.2 ; 14.3]$ & 0.79 \\
Hemoglobin, g/dL & $82.0[65.5 ; 118]$ & $76.0[48.0 ; 104]$ & 0.21 \\
Platelet $\left(\times 10^{9} / \mathrm{L}\right)$ & $45.3[31.3 ; 55.6]$ & $48.4[29.4 ; 59.5]$ & 0.58 \\
Donor age & $28.2[23.9 ; 32.8]$ & $26.8[22.8 ; 30.5]$ & 0.24 \\
Donor BMI & $4(11.8)$ & $12(15.4)$ & 0.77 \\
Donor diabetes mellitus, $n(\%)$ & $7(20.6)$ & $7(8.97)$ & 0.12 \\
DCD donor, $n$ (\%) & $390[320 ; 495]$ & $368[325 ; 483]$ & 0.52 \\
Cold ischemic time, min & &
\end{tabular}

DAA, direct-acting antiviral; LT, liver transplantation; MELD, model of end-stage liver disease; AFP, alphafetoprotein; DCD, donation after cardiac death; HCV, hepatitis C virus.

survival post-LT was compared between the 2 groups. We also performed a secondary analysis comparing HCC recurrence in the DAA group to patients who had achieved SVR12 after interferon-based treatment prior to LT (IFN group).

\section{Statistics}

Qualitative data were distributed by frequency and percentage and quantitative data were described by median (interquartile range). In the comparison between different subgroups, quantitative variables were compared using Wilcoxon tests. Categorical variables were compared using $\chi^{2}$ test or Fisher's exact tests, as appropriate. Time to HCC recurrence between groups was compared using Kaplan-Meier analysis with a log-rank test and with a Cox proportional hazards model in the multivariate setting. Patients were censored at last follow-up, graft failure, or death. The $p$ value $<0.05$ was considered statistically significant in all analyses. All analyses were performed in R version 3.4.3.

\section{Results}

\section{Baseline Characteristics}

A total of 151 patients underwent LT for HCC related to HCV: 34 patients in the DAA group, 95 patients in the HCV+ group, and 22 patients in the IFN group. At the time of LT, patients in the DAA group were older (60.2 vs. 57.2 years, $p=0.002$ ), had lower alpha-fetoprotein (AFP) ( $8.15 \mathrm{vs.} 22.3 \mathrm{ng} / \mathrm{mL}, p<0.01$ ), and had a lower calculated model for end-stage liver disease score ( 9 vs. $13, p<0.01$ ). All patients in the DAA group underwent deceased donor LT as compared to $82.1 \%$ in control group $(p=0.01)$. Patients in the DAA group spent 


\section{Tumors}

Table 2. DAA regimens

\begin{tabular}{l|l}
\hline DOI: $10.1159 / 000510341$ & $\begin{array}{l}\text { C } 2020 \text { The Author(s). Published by S. Karger AG, Basel } \\
\text { www.karger.com/gat }\end{array}$ \\
\hline
\end{tabular}

Lim et al.: DAAs and Recurrent HCC in Liver Transplant

\begin{tabular}{lc}
\hline DAA regimen & Frequency $(n)$ \\
\hline Sofosbuvir-ledipasvir & 16 \\
Sofosbuvir-ribavirin & 6 \\
Sofosbuvir-daclatasvir & 3 \\
Sofosbuvir-simeprevir & 8 \\
Ombitasvir-paritaprevir-ritonavir & 1 \\
\hline
\end{tabular}

DAA, direct-acting antiviral.

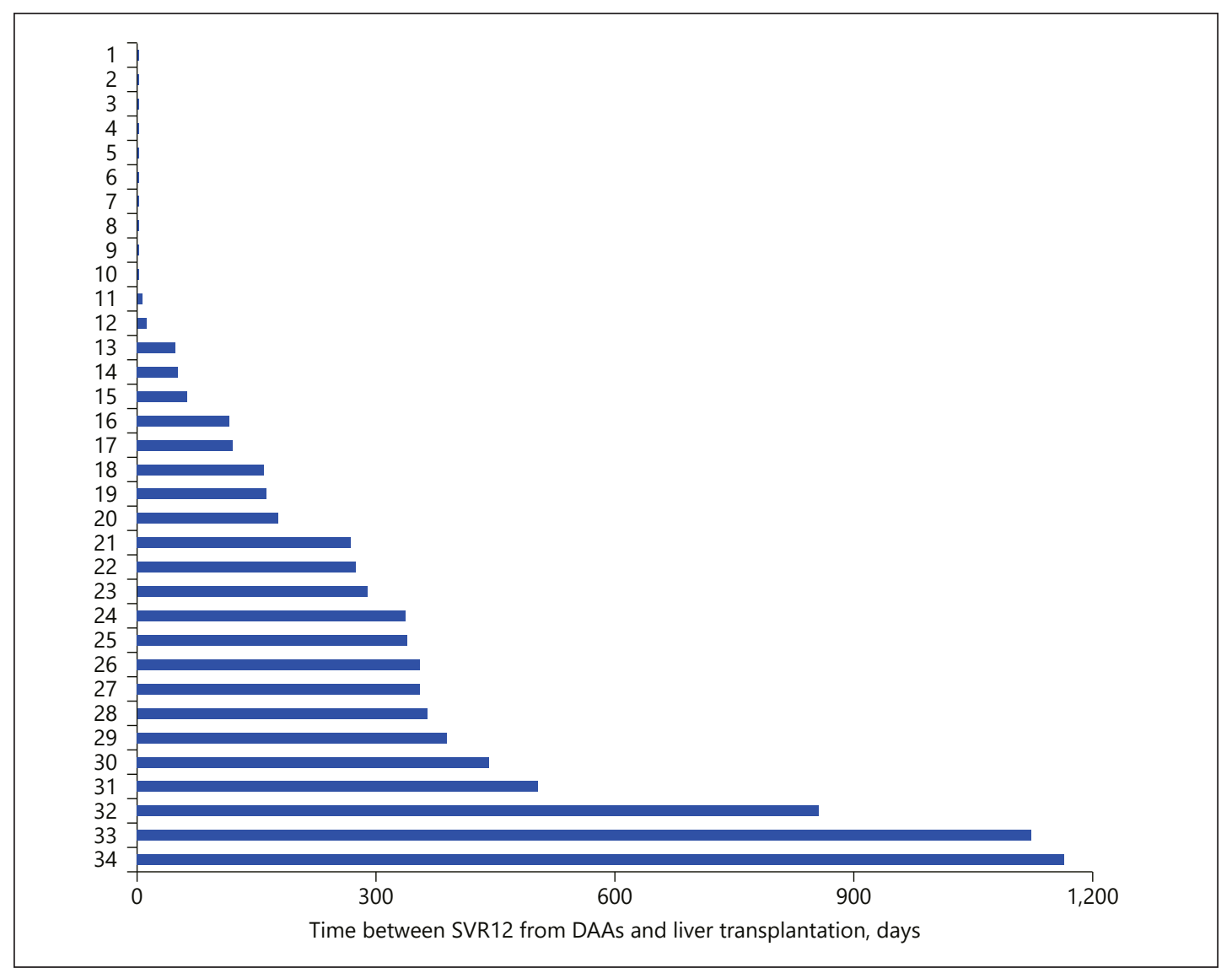

Fig. 1. Time between SVR12 and LT for individual patients achieving SVR with DAAs. DAA, direct-acting antiviral; LT, liver transplantation; SVR, sustained virological response.

longer time on the liver transplant waiting list compared to the control group (1.16 vs. 0.58 years, $p<0.01)$. There were no differences between the DAA group and the control group with regard to donor characteristics (Table 1).

Sofosbuvir-ledipasvir was the most common DAA regimen used pre-LT (10 patients, $29.4 \%$ ), with genotype 1 being the most common HCV genotype in both the groups (Table 2). The median time between SVR and LT in the DAA group was 139 days (interquartile range, 0-350 days) (Fig. 1).

Twenty-two patients achieved SVR with interferon-based therapy prior to LT. In comparison to the DAA group, a lower proportion of patients in the IFN group underwent deceased donor LT (81.8 vs. $100 \%, p=0.02$ ) and a higher proportion of patients had trans- 
Table 3. Tumor characteristics ( $n(\%)$ or median [IQR])

\begin{tabular}{lccc}
\hline & DAA group & HCV+ group & $p$ value \\
\hline $\begin{array}{l}\text { Pre-LT } \\
\text { Size at diagnosis, cm }\end{array}$ & $2.50[2.20 ; 3.10]$ & $2.50[1.95 ; 3.45]$ & 0.53 \\
No. of lesions at diagnosis, $n$ (\%) & $20(60.6)$ & $36(56.2)$ & \\
1 & $8(24.2)$ & $20(31.2)$ & 0.57 \\
2 & $4(12.1)$ & $8(12.5)$ & \\
3 & $1(3.03)$ & $0(0.00)$ & 0.08 \\
4 & $1.60[1.50 ; 2.10]$ & $2.00[1.60 ; 2.75]$ & \\
Size before transplant, cm & $17(50.0)$ & & \\
LRT pre-LT, $n$ (\%) & $5(14.7)$ & $11(76.2)$ & 0.01 \\
TACE & $7(20.6)$ & $2(2.41)$ & $<0.01$ \\
RFA & $11(32.4)$ & $2(2.38)$ & $<0.01$ \\
Radioembolization & & & \\
Microwave ablation & $21^{\mathrm{a}}(61.8)$ & $50(59.5)$ & 0.99 \\
Explant pathology, $n$ (\%) & $9(45.0)$ & $28(56.0)$ & \\
Viable tumor present & $5(25.0)$ & $8(16.0)$ & \\
No. of viable tumors, $n$ (\%) & $2(10.0)$ & $1(2.0)$ & 0.33 \\
1 & $1(5.0)$ & $1(2.0)$ & \\
2 & $3(15.0)$ & $12(24.0)$ & \\
3 & $2.90[1.72 ; 3.50]$ & $2.20[1.70 ; 3.35]$ & 0.61 \\
4 & $1(5.0)$ & $5(10.0)$ & 0.67 \\
$>4$ & $6(31.6)$ & $9(23.1)$ & \\
Largest tumor size, cm & $7(36.8)$ & $27(69.2)$ & 0.02 \\
Vascular invasion present, $n$ (\%) & $6(31.6)$ & $3(7.69)$ & \\
Differentiation of tumor, $n$ (\%) & & & \\
Well & & & \\
Moderate & & & \\
Poor & & & \\
\hline
\end{tabular}

DAA, direct-acting antiviral; LRT, locoregional therapy; LT, liver transplantation; RFA, radiofrequency ablation; TACE, trans-arterial chemoembolization; HCV, hepatitis C virus. ${ }^{\text {a }}$ Complete data unavailable on 1 patient.

arterial chemoembolization (TACE) ( 85 vs. $50 \%, p=0.02$ ). Time on the wait-list prior to LT was similar between the 2 groups ( 1.16 vs. 1.1 years, $p=0.07)$. There were no differences between the 2 groups with regard to donor characteristics (see online suppl. Table 1; for all online suppl. material, see www.karger.com/doi/10.1159/000510341).

\section{Tumor Characteristics}

There were no differences in the mean diameter of the largest HCC lesion or the mean number of lesions pre-LT between the 2 groups. Of note, 1 patient in the DAA group was successfully down-staged with LRT prior to listing for transplant. TACE was the most common liver-directed treatment for HCC pre-LT in both groups (Table 3). However, a higher proportion of patients underwent TACE in the HCV+ group (76.2 vs. 50\%, $p=0.01)$ and higher proportion underwent microwave ablation in the DAA group (32.4 vs. $2.38 \%, p<$ 0.01). There was no significant difference between the DAA group and HCV+ group for viable tumor on explant pathology (61.8 vs. $59.5 \%, p=0.99$ ). In patients with viable tumor on explant, there was a significant difference with regard to tumor differentiation but no differences with regard to number of viable tumors, largest tumor size, or presence of vascular invasion (Table 3). 
Gastrointestinal

Tumors

Fig. 2. HCC-free survival, DAA group versus $\mathrm{HCV}+$ group. DAA, LT patients with SVR12 from direct-acting agents prior to LT; $\mathrm{HCV}+$, LT patients with positive HCV RNA at LT. DAA, direct-acting antiviral; LT, liver transplantation; HCC, hepatocellular carcinoma; HCV, hepatitis $\mathrm{C}$ virus.

Table 4. Multivariate analysis for post-LT HCC recurrence

\begin{tabular}{l|l}
\hline Gastrointest Tumors 2020;7:134-143 \\
\hline DOI: 10.1159/000510341 & $\begin{array}{l}\text { @ } 2020 \text { The Author(s). Published by S. Karger AG, Basel } \\
\text { www.karger.com/gat }\end{array}$ \\
\hline
\end{tabular}

Lim et al.: DAAs and Recurrent HCC in Liver Transplant

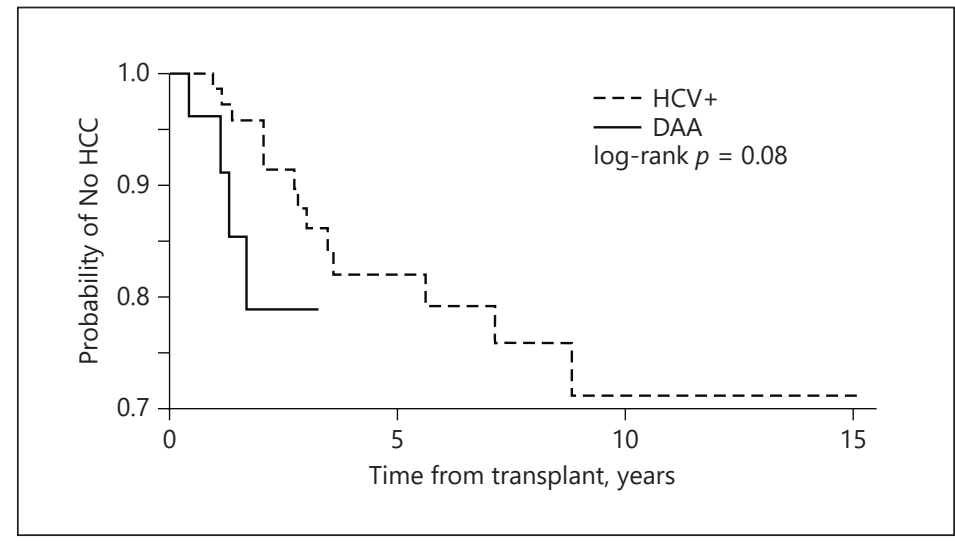

\begin{tabular}{lcl}
\hline Clinical variable & HR $(95 \%$ CI $)$ & $p$ value \\
\hline DAA use & $5.17(0.9,29.81)$ & 0.07 \\
Age at transplant & $0.97(0.86,1.10)$ & 0.6 \\
MELD score at transplant & $0.97(0.87,1.08)$ & 0.59 \\
Deceased donor LT & $0.25(0.05,1.23)$ & 0.09 \\
Time on the waiting list & $0.78(0.22,2.73)$ & 0.7 \\
Platelet & $1(0.78,1.25)$ & 0.93 \\
Albumin & $0.94(0.37,2.42)$ & 0.9 \\
AFP & $1(1,1)$ & 0.97 \\
TACE & $1.49(0.25,8.77)$ & 0.66 \\
Viable tumor on explant & $0.9(0.22,3.72)$ & 0.89 \\
\hline
\end{tabular}

DAA, direct-acting antiviral; MELD, model of end-stage liver disease; LT, liver transplant; AFP, alpha-fetoprotein; TACE, trans-arterial chemoembolization; HCC, hepatocellular carcinoma.

In the secondary analysis, a higher proportion of patients in the IFN group underwent TACE than those in the DAA group ( 85 vs. $50 \%, p=0.02$ ). There were no other significant differences for tumor characteristics between patients in the DAA group and the IFN group (online suppl. Table 2).

\section{Primary Outcome}

The Kaplan-Meier estimates of HCC-free survival were $96.2,96.2$, and $78.8 \%$ at 6,12 , and 24 months in the DAA group, respectively, and $100,98.6$, and $95.8 \%$ at 6,12 , and 24 months in the HCV+ group, respectively (Fig. 2). The difference in HCC-free survival between the 2 groups was not statistically significant (log-rank $p=0.08$ ).

In the secondary analysis, the estimates of HCC-free survival were 100, 90.2 , and $90.2 \%$ at 6,12, and 24 months in the IFN group. There was no difference in HCC-free survival between the DAA group and the IFN group (log-rank $p=0.45$ ) (online suppl. Fig. 1).

In a multivariate analysis adjusting for several factors, the impact of DAA use on HCC recurrence after LT did not reach statistical significance, but did show a strong trend (HR $5.17,95 \%$ CI 0.89-29.81, $p=0.07$ ). Deceased donor LT showed a trend toward decreased HCC recurrence after LT as compared to living donor LT (HR 0.25, 95\% CI 0.05-1.23, $p=0.09$ ). Age, model for end-stage liver disease score, time on the waiting list, platelet count, serum albumin, serum AFP, TACE, and the presence of viable tumor on explant pathology had no effect on HCC recurrence after LT (Table 4). 
Lim et al.: DAAs and Recurrent HCC in Liver Transplant

\section{Discussion}

There has been much debate regarding the role of DAAs in both the development and recurrence of HCC. Our retrospective cohort study showed a strong trend in reduced HCC-free survival in LT recipients who achieved SVR from DAAs prior to LT for HCC, compared to viremic controls. Although our secondary analysis was limited by low numbers, this difference did not exist when comparing LT recipients who achieved SVR from DAAs to those who achieved SVR from interferon-based treatment, suggesting a unique pathophysiology related to DAA use and post-LT HCC recurrence.

Furthermore, multivariate analysis showed that pre-LT SVR with DAAs increased the risk of HCC recurrence in LT recipients. Our model incorporated a number of factors associated with increased HCC recurrence, including laboratory abnormalities associated with decompensated liver disease, time on the waiting list, and posttransplant pathology. Of note, in our cohort, SVR with DAAs prior to LT was associated with HCC recurrence despite longer waitlist times than controls.

Although our results did not reach statistical significance, the potential clinical impact of our findings is high with a 5.17-fold increased relative risk of HCC recurrence. It is also important to emphasize that with a $p$ value of 0.07 , this result was very close to achieving the traditional definition of statistical significance, that is, 95\% confidence. Moreover, this was the primary outcome and not a secondary analysis.

The implementation of the Milan criteria for LT has revolutionized the management of HCC, but post-LT recurrence continues to be a problem today [2]. Recurrent HCC after LT is estimated between 11 and $18 \%$ and can be a devastating event [20]. Risk factors for post-LT HCC recurrence include elevated serum AFP prior to LT, vascular invasion of the tumor, poor tumor differentiation on histopathology, and history of prior hepatectomy [21, 22]. Data reported by the A2ALL study group have also shown that the risk of HCC recurrence is higher in patients undergoing living donor LT for HCC when than in deceased donor LT, presumably related to shorter times on the LT waiting list, which is consistent with the trend seen in our multivariate analysis $[23,24]$. Our study suggests that SVR with DAAs for HCV before LT could be an additional risk factor for HCC recurrence after LT.

Controversy currently exists regarding the role of DAAs in the development of HCC in patients with HCV-related cirrhosis. Our results concur with earlier reports raising the concern for the role of DAAs in the recurrence of HCC after treatment. The first study to link DAA use to recurrence of HCC showed that $27.6 \%$ of patients treated with DAAs unexpectedly developed early recurrent HCC following complete radiological response to various therapeutic interventions [15]. By contrast, a large retrospective study from France showed a low rate of HCC recurrence in patients treated with DAAs, and no difference between patients exposed to DAAs as compared to those who were not [25]. Recently, 2 large retrospective studies demonstrated no differences in the occurrence of HCC in patients treated with DAAs compared to those not receiving DAAs and a large multicenter study from North America drew a similar conclusion $[18,26,27]$.

Our study is the largest to examine recurrence of HCC in LT recipients undergoing treatment with DAAs prior to transplantation. Two studies have looked at the HCC recurrence in patients treated with DAAs and who have received LT. One brief report described a high proportion of early post-LT HCC recurrence in patients treated with DAAs prior to LT but was limited by a small sample size [19]. Another study reported a very low rate of HCC recurrence in LT recipients treated with DAAs after transplantation albeit a high proportion $(\sim 42 \%)$ of these patients presented with metastatic disease [25].

The primary theory postulated to explain why DAAs may increase HCC recurrence rates after SVR pertains to modification of immune surveillance posttreatment. HCV cure with inter- 
feron has been shown to reduce the incidence of HCC possibly by direct action against cancer cells or by stimulating the immune system to act against them [28]. DAAs may alter immunosurveillance of cancer cells due to a relatively abrupt change in the immune milieu with SVR [15]. Indeed, the absence of HCV has been shown to reduce the presence of NK cell-stimulating cytokines [29]. Another hypothesis is that there are now more patients at higher risk for the development of HCC (older patients and decompensated liver disease) undergoing treatment for HCV due to improved efficacy and reduced side effect profile of DAAs. These patients would not have undergone HCV treatment in the era of interferon-based therapy [30].

In LT recipients, it is possible that the risk of HCC recurrence after SVR from DAAs is higher as a result of the use of immunosuppression. Immunosuppression is associated with an increased risk of de novo malignancies in LT recipients with patients at particular risk for the development of skin and hematological cancers [31,32]. Several retrospective studies have implicated higher levels of immunosuppression in the development of recurrent HCC after LT [33-35]. The functional impairment of the immune system that results from immunosuppression in addition to reduced immunosurveillance of tumor cells following SVR with DAAs may provide the ideal environment for HCC recurrence in susceptible LT recipients.

Our study is unique in that we were able to apply appropriate survival analytics with patients censored at last follow-up, graft failure, or death because our center conducts lifetime follow-up on all LT patients, including documentation of malignancies. Other studies have only examined short-term recurrence. Moreover, our study contains extensive data on the tumor characteristics both before LT and upon examination of the explant. This study is also the first to examine the role of DAAs in the recurrence of HCC in patients undergoing microwave ablation. A notable proportion of our cohort received microwave ablation as LRT for HCC, significantly more in the DAA group, reflecting a culture change in management of early-stage HCC at our institution. Microwave ablation was first reported as a treatment for HCC in 1993 and is comparable to radiofrequency ablation with regard to cancer-free and overall survival [36-39].

Our study is limited by its retrospective, single-center nature. The relatively low numbers of patients in the secondary analysis made it difficult to draw any clear conclusions when comparing HCC recurrence in patients with SVR from DAA to interferon-based treatment. In addition, the relatively short follow-up of patients in the DAA group limits our ability to comment on long-term recurrence of HCC in patients treated with these agents.

In summary, we report a statistically insignificant but strong trend toward increased HCC recurrence when comparing LT recipients who achieved SVR with DAA therapy prior to transplantation to viremic controls, which we believe to be very clinically relevant. The decision to treat HCV prior to transplant remains a very individualized one that must balance the undoubtable benefits to post-LT graft function with access to hepatitis C-positive organs [40-42]. Until larger studies with long-term follow-up are performed, our results suggest that caution is still required when considering the use of DAAs to treat HCV in patients with HCC prior to LT.

\section{Acknowledgements}

We would like to acknowledge Dr. David Vock, associate professor of biostatistics, School of Public Health, University of Minnesota, for his invaluable advice regarding the statistical methods in the manuscript.

\section{Statement of Ethics}

This research was approved by the University of Minnesota Institutional Review Board (IRB Protocol \#0704M05324). Informed consent was not required due to the retrospective nature of the study. 


\section{Conflict of Interest Statement}

The authors have no conflicts of interest to declare.

\section{Funding Sources}

This study did not receive nor require any funding.

\section{Author Contributions}

N.L. designed the study, edited the manuscript, and provided critical mentoring to write the manuscript; D.S. acquired data, wrote the first draft of the manuscript, and prepared the figures and tables; S.J. did statistical analysis; and J.L. designed the study and critically revised the final draft of the manuscript.

\section{References}

1 Park JW, Chen M, Colombo M, Roberts LR, Schwartz M, Chen PJ, et al. Global patterns of hepatocellular carcinoma management from diagnosis to death: the BRIDGE study. Liver Int. 2015 Sep;35(9):2155-66.

2 Mazzaferro V, Regalia E, Doci R, Andreola S, Pulvirenti A, Bozzetti F, et al. Liver transplantation for the treatment of small hepatocellular carcinomas in patients with cirrhosis. N Engl J Med. 1996 Mar;334(11):693-9.

3 Kim WR, Lake JR, Smith JM, Skeans MA, Schladt DP, Edwards EB, et al. OPTN/SRTR 2015 annual data report: liver. Am J Transplant. 2018 Jan;17(Suppl 1):174-251.

4 De Martel C, Maucort-Boulch D, Plummer M, Franceschi S. World-wide relative contribution of hepatitis B and C viruses in hepatocellular carcinoma. Hepatology. 2015 Oct;62(4):1190-200.

5 Makarova-Rusher OV, Altekruse SF, McNeel TS, Ulahannan S, Duffy AG, Graubard BI, et al. Population attributable fractions of risk factors for hepatocellular carcinoma in the United States. Cancer. 2016 Jun 1;122(11): 1757-65.

6 Udompap P, Mannalithara A, Heo NY, Kim D, Kim WR. Increasing prevalence of cirrhosis among U.S. adults aware or unaware of their chronic hepatitis C virus infection. J Hepatol. 2016 May;64(5):1027-32.

7 Afdhal N, Zeuzem S, Kwo P, Chojkier M, Gitlin N, Puoti M, et al. Ledipasvir and sofosbuvir for untreated HCV genotype 1 infection. N Engl J Med. 2014 May;370(20):1889-98.

8 Sulkowski MS, Gardiner DF, Rodriguez-Torres M, Reddy KR, Hassanein T, Jacobson I, et al. Daclatasvir plus sofosbuvir for previously treated or untreated chronic HCV infection. N Engl J Med. 2014 Jan;370(3):211-21.

9 Charlton M, Everson GT, Flamm SL, Kumar P, Landis C, Brown RS, et al. Ledipasvir and Sofosbuvir plus ribavirin for treatment of HCV infection in patients wth advanced liver disease. Gastroenterology. 2015 Sep; 149(3):649-59.

10 Foster GR, Afdhal N, Roberts SK, Bräu N, Gane EJ, Pianko S, et al. Sofosbuvir and velpatasvir for HCV genotype 2 and 3 infection. N Engl J Med. 2015 Dec;373(27):2608-17.

11 Gane EJ, Agarwal K. Directly acting antivirals (DAAs) for the treatment of chronic hepatitis C virus infection in liver transplant patients: "a flood of opportunity". Am J Transplant. 2014 May;14(5):994-1002.

12 Curry MP, Forns X, Chung RT, Terrault NA, Brown R, Fenkel JM, et al. Sofosbuvir and ribavirin prevent recurrence of HCV infection after liver transplantation: an open-label study. Gastroenterology. 2015 Jan;148(1): 100-e1.

13 Chen T, Terrault NA. Perspectives on treating hepatitis C infection in the liver transplantation setting. Curr Opin Organ Transplant. 2016 Apr;21(2):111-9.

14 Conti F, Buonfiglioli F, Scuteri A, Crespi C, Bolondi L, Caraceni P, et al. Early occurrence and recurrence of hepatocellular carcinoma in HCV-related cirrhosis treated with direct-acting antivirals. J Hepatol. 2016 0ct;65(4): 727-33.

15 Reig M, Mariño Z, Perelló C, Iñarrairaegui M, Ribeiro A, Lens S, et al. Unexpected high rate of early tumor recurrence in patients with HCV-related HCC undergoing interferon-free therapy. J Hepatol. 2016 Oct;65(4):71926.

16 Zanetto A, Shalaby S, Vitale A, Mescoli C, Ferrarese A, Gambato M, et al. Dropout rate from the liver transplant waiting list because of hepatocellular carcinoma progression in hepatitis $\mathrm{C}$ virus-infected patients treated with direct-acting antivirals. Liver Transpl. 2017 Sep;23(9):1103-12.

17 Huang AC, Mehta N, Dodge JL, Yao FY, Terrault NA. Direct-acting antivirals do not increase the risk of hepatocellular carcinoma recurrence after local-regional therapy or liver transplant waitlist dropout. Hepatology. 2018 Aug;68(2):449-61.

18 Singal AG, Rich NE, Mehta N, Branch A, Pillai A, Hoteit M, et al. Direct-acting antiviral therapy not associated with recurrence of hepatocellular carcinoma in a multicenter North American cohort study. Gastroenterology. 2019 May;156(6):1683-92. 
19 Yang JD, Aqel BA, Pungpapong S, Gores GJ, Roberts LR, Leise MD. Direct acting antiviral therapy and tumor recurrence after liver transplantation for hepatitis C-associated hepatocellular carcinoma. J Hepatol. 2016 Oct;65(4):859-60.

20 Halazun KJ, Najjar M, Abdelmessih RM, Samstein B, Griesemer AD, Guarrera JV, et al. Recurrence after liver transplantation for hepatocellular carcinoma: a new MORAL to the story. Ann Surg. 2017 Mar;265(3):557-64

21 Escartin A, Sapisochin G, Bilbao I, Vilallonga R, Bueno J, Castells L, et al. Recurrence of hepatocellular carcinoma after liver transplantation. Transplant Proc. 2007 Sep;39(7):2308-10.

22 Zimmerman MA, Ghobrial RM, Tong MJ, Hiatt JR, Cameron AM, Hong J, et al. Recurrence of hepatocellular carcinoma following liver transplantation: a review of preoperative and postoperative prognostic indicators. Arch Surg. 2008 Feb;143(2):182-8;

23 Fisher RA, Kulik LM, Freise CE, Lok AS, Shearon TH, Brown RS, et al. Hepatocellular carcinoma recurrence and death following living and deceased donor liver transplantation. Am J Transplant. 2007 Jun;7(6):1601-8.

24 Kulik LM, Fisher RA, Rodrigo DR, Brown RS, Freise CE, Shaked A, et al. Outcomes of living and deceased donor liver transplant recipients with hepatocellular carcinoma: results of the A2ALL cohort. Am J Transplant. 2012 Nov;12(11):2997-3007.

25 ANRS Collaborative Study Group on Hepatocellular Carcinoma (ANRS C022 HEPATHER, C012 CirVir and CO23 CUPILT cohorts). Lack of evidence of an effect of direct-acting antivirals on the recurrence of hepatocellular carcinoma: data from three ANRS cohorts. J Hepatol. 2016 Oct;65(4):734-40.

26 Kanwal F, Kramer J, Asch SM, Chayanupatkul M, Cao Y, El-Serag HB. Risk of hepatocellular cancer in HCV patients treated with direct-acting antiviral agents. Gastroenterology. 2017 Oct;153(4):996-1005.e1.

27 Calvaruso V, Cabibbo G, Cacciola I, Petta S, Madonia S, Bellia A, et al. Incidence of hepatocellular carcinoma in patients with HCV-associated cirrhosis treated with direct-acting antiviral agents. Gastroenterology. 2018 Aug;155(2):411-21.e1.

28 Miyake Y, Takaki A, Iwasaki Y, Yamamoto K. Meta-analysis: interferon-alpha prevents the recurrence after curative treatment of hepatitis C virus-related hepatocellular carcinoma. J Viral Hepat. 2010 Apr;17(4):287-92.

29 Spaan M, van Oord G, Kreefft K, Hou J, Hansen BE, Janssen HL, et al. Immunological analysis during interferonfree therapy for chronic hepatitis $\mathrm{C}$ virus infection reveals modulation of the natural killer cell compartment. J Infect Dis. 2016 Jan;213(2):216-23.

30 Innes H, Barclay ST, Hayes PC, Fraser A, Dillon JF, Stanley A, et al. The risk of hepatocellular carcinoma in cirrhotic patients with hepatitis $\mathrm{C}$ and sustained viral response: role of the treatment regimen. J Hepatol. 2018 Apr;68(4):646-54.

31 Penn I. Posttransplantation de novo tumors in liver allograft recipients. Liver Transpl Surg. 1996 Jan;2(1): 52-9.

32 Benlloch S, Berenguer M, Prieto M, Moreno R, San Juan F, Rayón M, et al. De novo internal neoplasms after liver transplantation: increased risk and aggressive behavior in recent years? Am J Transplant. 2004 Apr;4(4): 596-604.

33 Vivarelli M, Cucchetti A, La Barba G, Ravaioli M, Del Gaudio M, Lauro A, et al. Liver transplantation for hepatocellular carcinoma under calcineurin inhibitors: reassessment of risk factors for tumor recurrence. Ann Surg. 2008 Nov;248(5):857-62.

34 Vivarelli M, Dazzi A, Zanello M, Cucchetti A, Cescon M, Ravaioli M, et al. Effect of different immunosuppressive schedules on recurrence-free survival after liver transplantation for hepatocellular carcinoma. Transplantation. 2010 Jan;89(2):227-31.

35 Rodríguez-Perálvarez M, Tsochatzis E, Naveas MC, Pieri G, García-Caparrós C, O’Beirne J, et al. Reduced exposure to calcineurin inhibitors early after liver transplantation prevents recurrence of hepatocellular carcinoma. J Hepatol. 2013 Dec;59(6):1193-9.

36 Saitsu H, Mada Y, Taniwaki S, Okuda K, Nakayama T, Oishi K, et al. [Investigation of microwave coagulonecrotic therapy for 21 patients with small hepatocellular carcinoma less than $5 \mathrm{~cm}$ in diameter]. Nihon Geka Gakkai Zasshi. 1993 Apr;94(4):359-65.

37 Sato M, Watanabe Y, Ueda S, Iseki S, Abe Y, Sato N, et al. Microwave coagulation therapy for hepatocellular carcinoma. Gastroenterology. 1996 May;110(5):1507-14.

38 Facciorusso A, Di Maso M, Muscatiello N. Microwave ablation versus radiofrequency ablation for the treatment of hepatocellular carcinoma: a systematic review and meta-analysis. Int J Hyperthermia. 2016 May;32(3): 339-44.

39 Santambrogio R, Chiang J, Barabino M, Meloni FM, Bertolini E, Melchiorre F, et al. Comparison of laparoscopic microwave to radiofrequency ablation of small hepatocellular carcinoma $(\leq 3 \mathrm{~cm})$. Ann Surg Oncol. 2017 Jan; 24(1):257-63.

40 Carrión JA, Navasa M, García-Retortillo M, García-Pagan JC, Crespo G, Bruguera M, et al. Efficacy of antiviral therapy on hepatitis $\mathrm{C}$ recurrence after liver transplantation: a randomized controlled study. Gastroenterology. 2007 May;132(5):1746-56.

41 Veldt BJ, Poterucha JJ, Watt KD, Wiesner RH, Hay JE, Kremers WK, et al. Impact of pegylated interferon and ribavirin treatment on graft survival in liver transplant patients with recurrent hepatitis C infection. Am J Transplant. 2008 Nov;8(11):2426-33.

42 Fortune BE, Martinez-Camacho A, Kreidler S, Gralla J, Everson GT. Post-transplant survival is improved for hepatitis $\mathrm{C}$ recipients who are RNA negative at time of liver transplantation. Transpl Int. 2015 Aug;28(8): 980-9. 